\title{
Solar wind vs magnetosheath turbulence and Alfvén vortices
}

\section{O. Alexandrova}

LESIA, Observatoire de Paris, CNRS, UPMC, Université Paris Diderot; 5 Place Jules Janssen, 92190 Meudon, France

now at: Institute of Geophysics and Meteorology, University of Cologne, Germany

Received: 11 May 2007 - Revised: 23 October 2007 - Accepted: 4 January 2008 - Published: 11 February 2008

\begin{abstract}
In this paper we give firstly a broad review of the space plasma turbulence around the ion characteristic space and temporal scales within two natural laboratories, the solar wind and the Earth magnetosheath. In both regions power law spectra of magnetic fluctuations are observed. In both regions these spectra have a break in the vicinity of the ion cyclotron frequency. A distinctive feature of the magnetosheath turbulence is the presence of Alfvén vortices at scales of the spectral break. The Alfvén vortices are multi-scale nonlinear structures. We give a review of the main theoretical features of incompressible Alfvén vortsices in the second part of the paper. Finally, we analyze the spectral properties of the Alfvén vortex solution and of the network of such vortices. We show that the observed magnetosheath spectrum in presence of the Alfvén vortices can be described, at least partially, by the vortex network model.
\end{abstract}

\section{Introduction}

Natural plasmas are frequently in a turbulent state. Turbulence is a non-linear process, non-reproducible locally but with some universal statistical properties. To date, there is no analytical theory that describes 3-D fluid turbulence in a sufficiently general frame. At the same time, thanks to a number of observations, numerical simulations and theoretical works, we know these universal properties of a turbulent system:

- In Fourier space, the energy of turbulent fluctuations is distributed as a power law. This reflects a scale invariance, i.e., at each scale the same physical description is valid.

Correspondence to: O. Alexandrova

(olga.alexandrova@obspm.fr)
- Intermittency (spatial inhomogeneity of the turbulence), that manifests itself as non-Gaussian probability distribution functions of turbulent fluctuations, and dependence of this non-Gaussianity on scale.

In 3-D incompressible homogeneous non-magnetized fluids, when the energy injection scale is far from the dissipation scale, on the intermediate scales (inertial range) the power spectrum of velocity fluctuations follows a $k^{-s}$ law with $s=5 / 3$. This law is well described by the Kolomogorov's phenomenology and depends neither on the energy injection nor on the energy dissipation processes (Frisch, 1995).

Intermittency is beyond this phenomenology but we know that in hydrodynamics it appears in the form of coherent structures as filaments of vorticity. Their characteristic length is of the order of the energy injection scale but their diameter is of the order of the dissipation scale (see the references of section 8.9 in (Frisch, 1995)). Thus, in Fourier space, these filaments occupy the edges of the inertial range.

In neutral fluids the dissipation sets in at scales of the order of the collisional mean free path. At these scales the fluctuation energy spectrum is also universal, but with an exponential shape and not a power law (Frisch, 1995).

The solar wind and the Earth's magnetosheath may serve as laboratories for the study of turbulence in a collisionless, moderately magnetized plasma due to their relative proximity and the possibility to study them with in-situ measurements. In both cases, the collisional mean free path is of the order of the Sun-Earth distance and so dissipation via collisions is negligible. At the same time, at the difference with neutral fluids, in magnetized plasma there is a number of characteristic (microscopic) space and temporal scales. The solar wind is an example of freely developed turbulence, the Earth magnetosheath on the contrary is a domain bounded by the bow-shock and the magnetopause, where there is an important energy injection at the ion scales. By studying

Published by Copernicus Publications on behalf of the European Geosciences Union and the American Geophysical Union. 
the magnetohydrodynamic (MHD) turbulence in such different laboratories, we can improve our understanding of the plasma turbulence in other astrophysical situations, such as the interstellar medium or the supernova remnants, for example.

In this paper we firstly give a broad review of the space plasma turbulence around the ion characteristic space and temporal scales in the solar wind and in the Earth's magnetosheath. Secondly we give a review of the main theoretical features of incompressible Alfvén vortices, coherent structures observed in the magnetosheath. Finally we analyze in details the spectral properties of the vortices.

\subsection{Solar wind}

The solar wind is the extension of the solar atmosphere expanding into the interplanetary space. The expansion speed depends on the topology of the solar magnetic field: the fast solar wind blows along open field lines, while the slow wind escapes from the regions with closed ones. The discussion presented in this section is valid only for one type solar wind, fast or slow, not for the mixture of both, and at $1 \mathrm{AU}^{1}$.

The fast and slow solar wind flows are filled with magnetic fluctuations. A considerable number of studies have been dedicated to the analysis of the magnetic fluctuations on the magnetohydrodynamical (MHD) scales, see for example the following review papers (Tu and Marsch, 1995; Bruno and Carbone, 2005; Horbury et al., 2005). One of the most important results is the presence of large scale Alfvén waves, originating in the solar corona and propagating away from the Sun (Belcher and Davis, 1971). The superposition of these waves forms a $\sim f^{-1}$ spectrum at frequencies below $10^{-4} \mathrm{~Hz}$. At higher frequencies the spectrum follows $\mathrm{a} \sim f^{-5 / 3}$ power law, that is usually interpreted in terms of an active turbulent cascade, which transfers energy from the Alfvén waves at large scales up to the ion scales ${ }^{2}$. This range, dominated by Alfvénic fluctuations extends from $\sim 10^{-4} \mathrm{~Hz}$ to $\sim 10^{-1} \mathrm{~Hz}$. Before discussing more in details what is going on at higher frequencies, some considerations about the Alfvénic cascade are in order.

First, one may wonder wether the Alfvénic cascade results from the superposition of almost linear plasma waves or of definitely non-linear fluctuations. A mixture of weakly interacting waves is known as weak turbulence: the life time of the waves is much longer than the energy exchange time,

\footnotetext{
${ }^{1}$ The statistical properties of the large-scale magnetic fluctuations (at time scales larger than a day) as a function of distance from the Sun between 1 to $60 \mathrm{AU}$ are described in (Burlaga et al., 2003).

${ }^{2} \mathrm{~A}$ discussion about small scale discontinuities (on kinetic scales) induced by the steepening of large scale Alfvén waves, with wavelengths $\sim 0.01 \mathrm{AU}$, can be found in (Tsurutani et al., 2005); a possible relationship between the small scale solar wind discontinuities and the Alfvénic turbulent fluctuations has been recently reported in (Vasquez et al., 2007).
}

and there are no phase correlations between the waves. On the contrary, the turbulence described by a Kolmogorov-like phenomenology refers to strong turbulence, where the fluctuations exchange energy in times of the order of their life time.

The observation of the $f^{-5 / 3}$ spectrum of magnetic fluctuations independently on the solar wind type and local plasma parameters (Leamon et al., 1998; Smith et al., 2006) indicates that the Kolmogorov description seems to be more appropriate. In fact, thanks to the superalfvénic speed of the solar wind $V$, the turbulent fluctuations can be considered frozen in the flow and so the observed variations on time scale $\delta t$ correspond to variations on spatial scale $\delta r=V \delta t$ (Taylor hypothesis). Hence, the observed spectrum $f^{-5 / 3}$ within the frequency range $\left[10^{-4}, 10^{-1}\right] \mathrm{Hz}$ corresponds to $E \sim k^{-5 / 3}$ for the space scales $\left[10^{4}, 10^{7}\right] \mathrm{km}$.

Another important argument in favor of the strong turbulence description is intermittency. It was shown that at time scales where the $k^{-5 / 3}$-spectrum is observed the probability distribution functions (PDFs) of magnetic fluctuations behaves as velocity increments in strong hydrodynamic turbulence (Sorriso-Valvo et al., 1999), i.e. the smaller the scale the higher PDF's tails and the larger deviation from the Gaussian statistics. The power-law index and intermittency are important arguments in favor of the strong turbulence description of the solar wind fluctuations. However, to confirm that, estimations of the life time of the fluctuations in comparison with the energy exchange time should be done.

Second, even if Kolmogorov's spectrum is observed in the solar wind, there are important differences with turbulence in neutral fluids. In a plasma, the presence of a mean magnetic field $\mathbf{B}_{0}$ gives rise to anisotropy with respect to the field direction both in amplitudes of the fluctuations $\left(\delta B_{\perp}>\delta B_{\|}\right)$ and in their spatial structure $\left(k_{\perp}>k_{\|}\right)$, a signature of quasibidimensional turbulence with a non-linear transfer essentially perpendicular to $\mathbf{B}_{0}$ (Shebalin et al., 1983; Grappin, 1986; Bieber et al., 1996; Matthaeus et al., 1990, 1996; Müller and Grappin, 2005; Bruno and Carbone, 2005; Horbury et al., 2005; Dmitruk et al., 2005; Osman and Horbury, 2007).

The role of $\mathbf{B}_{0}$ is clearly seen in the "organization" of the intermittent structures of the solar wind turbulence. As shown by Veltri and Mangeney (1999) and Veltri (1999), on time scales of the order of few minutes the intermittent events take the form of (i) current sheets with the maximal field variation in the plane perpendicular to $\mathbf{B}_{0}$, or (ii) shock waves with normals along $\mathbf{B}_{0}$. Note that these structures are one dimensional, at variance with ordinary fluid intermittency, where the intermittent structures are two dimensional vortices, as we have discussed above ${ }^{3}$.

\footnotetext{
${ }^{3}$ We define the dimension of coherent structures as three minus the dimension of the symmetry group of the structure. For example, a cylindrical structure is an invariant under translation along one dimension (along its axis), and variations are important only in two
} 
Third, as already mentioned above, a specific feature of most of space plasmas is the unimportance of collisional dissipation and presence of a number of characteristic space and temporal scales, associated with significant changes in the dispersive properties of the medium. The question is therefore: what happens to turbulent magnetic fluctuations observed at space scales around the ions inertial length $c / \omega_{p i}$ ( $c$ being the speed of light and $\omega_{p i}$ - the ion plasma frequency) and at time scales around the ion cyclotron frequency $f_{c i}=e B_{0} / m_{i}$, (where $B_{0}$ is the mean field modulus, $e$ being the electron charge and $m_{i}$ is the ion mass)? The remaining part of this section will be devoted to a discussion of the possible answers to this question.

Observationally, the Kolmogorov-like inertial range ends in the vicinity of $f_{c i} \sim 0.1 \mathrm{~Hz}$, where the power spectrum of the magnetic fluctuations has a break ( $f_{c i}$ is calculated using the mean field at $\left.1 \mathrm{AU}, B_{0} \sim 5 \mathrm{nT}\right)$. Above the break point the spectrum has a significant steepening, but it is still described by a power law $f^{-s}$, with $s \in[2,4]$ (Leamon et al., 1998; Bale et al., 2005; Smith et al., 2006). Some authors associate the spectral steepening to the dissipation range (Leamon et al., 1998, 1999; Smith et al., 2006). Others suggest that beyond the spectral break another turbulent cascade takes place (Biskamp et al., 1996; Ghosh et al., 1996; Stawicki et al., 2001; Galtier, 2006; Galtier and Buchlin, 2007).

It is important to note that there are no direct observational evidence for the dissipation of the turbulent fluctuations and the association of the high frequency range with the dissipation range is just a hypothesis. This hypothesis can be studied numerically: Li et al. (2001) show, for example, that if the spectral break is associated to dissipation via kinetic damping of the Alfvén and magnetosonic waves, the spectrum would have been exponential and not a power-law.

As mentioned above, within the Kolmogorov-like inertial range (below the break) the solar wind magnetic fluctuations are mostly Alfvénic and nearly incompressible (Bruno and Carbone, 2005; Tu and Marsch, 1995). The ion cyclotron frequency is a cut-off for the left-handed Alfvénic fluctuations. Therefore, the spectral break in the vicinity of $f_{c i}$ represents a change in the nature of magnetic fluctuations. Galtier (2006) proposes to describe the spectrum above the break in the incompressible Hall MHD weak turbulence approximation, while Bale et al. (2005) suggest that the spectral break frequency corresponds to the Doppler-shifted ion Larmor radius and propose to explain the above spectrum by a mixture of kinetic Alfvén waves. However the applicability of the weak turbulence approximation (mixture of linear waves with random phases) to the solar wind turbulence is questionable, as we have discussed above. Furthermore, the incompressible approximation, well satisfied below the spectral break, is hardly applicable above it, since nearly $30 \%$ of

other dimensions, we call it 2-D structure (3-1). A plane structure, like a current sheet or a shock, is invariant in the plane, so their dimension is $(3-2)$. the fluctuations are compressible in this range (Alexandrova et al., 2008).

Recently, we have proposed some arguments in favor of a strong non-linear turbulent cascade rather than a dissipative or dispersive process to explain the form of the spectrum in the frequency domain above the spectral break (Alexandrova et al., 2007, 2008). We have shown in particular that in this range the intermittency increases toward small scales, similarly to what happens in the Kolmogorov-like inertial range.

Both the presence of a power-law spectrum and the intermittency increase towards small scales seem to be quite in contrast with what one would expect from dissipation. In usual fluid turbulence, the dissipative range is described by an exponential function and not by a power-law, as discussed above. In the near dissipation range (Chevillard et al., 2005) the intermittency increases exponentially as long as the Gaussian fluctuations dissipate faster than the coherent structures (i.e., there are mostly coherent structures in this range); then the fluctuations become self-similar, the singularities being smoothed by dissipation. In the solar wind turbulence we observe a completely different picture. The Kolmogorov inertial range is characterized by an increase of intermittency. At a spectral break point the intermittency stops growing, probably there is a partial destruction of coherent structures due to some local kinetic effects related to the ions (this point is worth studding in more details). Then, at smaller scales, the intermittency increases as a power law indicating that non-linear interactions are again at work to build up a new inertial range. The strong coherent structures represent only (2-6)\% of the fluctuations at each scale involved in this small-scale intermittent cascade (Alexandrova et al., 2008).

To describe the small scale cascade of the solar wind turbulence we have proposed a simple phenomenological model based on the compressible Hall MHD (Alexandrova et al., 2008). The phenomenological arguments are based on the fact that on the scales of the order of the ion inertial length $c / \omega_{p i}$ and at frequencies of the order of $f_{c i}$, the Hall term in the Faraday's law, proportional to $\nabla \times[(\mathbf{J} \times \mathbf{B}) / \rho]$, dominates. This means that the nonlinear energy transfer is realized in a time associated to the Hall effect $\tau_{H} \sim \rho_{\ell} \ell^{2} / B_{\ell}$, that must be shorter than the eddy-turnover time $\tau_{N L} \sim \ell / u_{\ell}$, here $\rho_{\ell}$ is the typical value of density, $u_{\ell}$ the velocity and $B_{\ell}$ the magnetic intensity for an eddy at length-scale $\ell$.

In a compressible fluid the energy balance equation must be expressed in terms of energy densities (i.e., energy per unit volume) and not in terms of specific energy (i.e., per unit mass), in order to take into account density fluctuations (see Fleck, 1996, for example). The mean volume rate of energy transfer on Hall times in a compressible fluid is therefore $\varepsilon_{V} \sim \rho_{\ell} u_{\ell}^{2} / \tau_{H}$. Assuming equipartition between kinetic and magnetic energies $\rho_{\ell} u_{\ell}^{2} \sim B_{\ell}^{2}$, the energy-transfer rate results to be proportional to $\varepsilon_{V} \sim B_{\ell}^{3} / \ell^{2} \rho_{\ell}$. Following von Weizsäcker (1951), the density scaling is $\rho_{\ell} \sim \ell^{-3 \gamma}$, where 
$|\gamma|$ is a measure of the degree of compression at each scale, and ranges from 0 for no compression up to 1 for isotropic compression $(3|\gamma|$ is a number of dimensions in which the compression takes place). Assuming now a constant spectrum energy transfer rate we have $B_{\ell} \sim \rho^{1 / 3} \ell^{2 / 3} \sim \ell^{2 / 3-\gamma}$. Therefore, the spectral energy function for the compressible fluctuations, which exchange their energy in Hall times, is

$E(k) \sim \frac{B_{\ell}^{2}}{k} \sim k^{-7 / 3+2 \gamma}$.

This phenomenological model allows to explain the variations of the spectral index of the high frequency part of the solar wind spectrum from -4 to -2 by different degree of plasma compression, $\gamma \in[-5 / 6,1 / 6]$, and without any dissipation. In the incompressible case $(\gamma=0)$ the well known $k^{-7 / 3}$-law for Electron MHD turbulence (Biskamp et al., 1996) is recovered. This simple model however must be improved in order to take into account (i) the space anisotropy that appears in a plasma with a mean field, and (ii) a possible different scalings of velocity and magnetic field that yields to an imbalance between kinetic and magnetic energies at small scales. Besides, the interpretation of the small scale energy cascade in the Hall MHD frame is supported by the observations of a clear correlation between the spectral break frequency and the Doppler-shifted wavevector of the ion inertia length $c / \omega_{p i}$ (Leamon et al., 2000).

The inquisitive reader can then ask "where is finally the dissipation range of the solar wind turbulence?", because some dissipative process must be at work. The observations on the electron scales, like the electron inertia length $\lambda_{e}$, and on the electrostatic scales close to the Debay's length $\lambda_{D}$ are crucial to answer this question. To my knowledge, there are no observations of the solar wind spectrum on such small scales.

\subsection{The Earth magnetosheath}

The magnetosheath turbulence is another example of MHD turbulence, although less studied. Here, the mean magnetic field is stronger than in the solar wind, the plasma is denser and hotter. The presence of boundaries, the Earth bow-shock and the magnetopause, can play a crucial role in the organization of plasma turbulence in this region. The shock geometry with respect to the interplanetary magnetic field $\mathbf{B}_{\mathrm{IMF}}$ determines the level and nature of the downstream turbulence. If the angle between the shock normal $\mathbf{n}$ and $\mathbf{B}_{\mathrm{IMF}}$ is small (quasi parallel shocks), $\Theta_{\mathrm{BN}} \in(0,45)^{\circ}$, the fluctuations from the solar wind are amplified and modified within the quasi-parallel shock front and penetrate downstream. In this case the level of magnetic fluctuations in the magnetosheath is rather high, $\delta B / B_{0} \sim 1$. When the angle between $\mathbf{n}$ and $\mathbf{B}_{\mathrm{IMF}}$ is large, (quasi-perpendicular shocks), $\Theta_{\mathrm{BN}} \in$ $(45,90)^{\circ}$, the downstream fluctuations are generated mainly locally and their amplitude is much smaller $\delta B / B_{0} \sim 0.1$.
A notable feature of the magnetosheath downstream of the quasi-perpendicular bow-shock is the ion temperature anisotropy that takes its origin in the vicinity of the bowshock and the magnetopause: the ion temperature perpendicular to the mean magnetic field $\left(T_{\perp}\right)$ is generally larger than the parallel one $\left(T_{\|}\right)$contrary to what is observed in the solar wind where $T_{\|}>T_{\perp}$, see for example (Hellinger et al., 2006; Matteini et al., 2006). This anisotropy is a source of free energy and its liberation proceeds through the generation of low frequency waves, i.e. at frequencies below $f_{c i}$. For the magnetosheath conditions $\left(T_{\perp}>T_{\|}\right)$the linear Vlasov theory for homogeneous plasmas predicts that if the ion plasma beta $\beta=8 \pi p / B_{0}^{2}$, the ratio between the pressure of the ions $p$ and the magnetic pressure, is small $(\beta \leq 1)$ Alfvén Ion Cyclotron (AIC) waves will grow with wave vectors mainly parallel to the background field $k_{\|} \gg k_{\perp}$, while for a high beta $(\beta \geq 5)$ mirror waves with $k_{\perp} \gg k_{\|}$will grow (Schwartz et al., 1996; Gary et al., 1994b). These waves then scatter and diffuse the particles, reducing the anisotropy. Most of previous works on the magnetosheath were devoted to the identification of AIC and mirror waves (Tsurutani et al., 1982; Lacombe et al., 1992; Anderson et al., 1994; Lucek et al., 2001; Sahraoui et al., 2003b; Alexandrova et al., 2004) to confirm this scenario of anisotropy relaxation. Very convincing results have been obtained, where one can see that AIC and Mirror instabilities control the magnetosheath state. These results are reviewed and discussed by Lacombe and Belmont (1995); similar results are obtained in numerical simulations such as those described by Hellinger et al. (2003).

Thus a simple quasi-linear theory can explain the lowfrequency fluctuations $\left(f<f_{c i}\right)$ in the magnetosheath. It is important to note, however, that monochromatic AIC and mirror modes, visible in Fourier spectrum as sharp peaks (see for example (Alexandrova et al., 2004)), are not frequently seen in the magnetosheath. Instead, most of the observations shows a broad band spectrum covering frequencies much higher than $f_{c i}$ (Siscoe et al., 1967; Rezeau et al., 1986, 1999, 2001; Matsuoka et al., 2000; Shevyrev and Zastenker, 2005; Sahraoui et al., 2003a, 2006). Let us discuss more in details some features of magnetic fluctuations below and above $f_{c i}$

A detailed analysis of magnetic power spectra downstream of a quasi-perpendicular shock for $f<f_{c i}$ has been made by Anderson et al. (1994). The authors subdivide the observed samples into groups of different $\beta$ and temperature anisotropy. They observe that when $\beta$ decreases $\left(T_{\perp} / T_{\|}\right.$increases) the spectra become more and more anisotropic in the sense of magnitude of the fluctuations: Alfvénic fluctuations dominate and cover a larger and larger frequency range. Let us develop this point in details.

It was observed (Anderson et al., 1994) that in the magnetosheath with $\beta \sim 7$ and the anisotropy $T_{\perp} / T_{\|} \sim 1.4$ mirror fluctuations are dominant up to $0.25 f_{c i}$. Between $0.25 f_{c i}$ and $f_{c i}$ nearly isotropic turbulence is observed, i.e. the amplitude of magnetic field fluctuations is the same along any 
direction. For a lower beta $\beta \sim 3\left(T_{\perp} / T_{\|} \sim 1.6\right)$ the magnetosheath turbulence becomes anisotropic with respect to the mean magnetic field: the Alfvénic fluctuations become dominant above $\sim 0.2 f_{c i}$. These fluctuations, however, are isotropic in the plane perpendicular to the mean field, i.e. the left-hand (LH) and right-hand ( $\mathrm{RH})$ polarized Alfvénic fluctuations have the same amplitudes. For $\beta<1.7$ and $T_{\perp} / T_{\|}>1.9$ Alfvénic fluctuations dominate the whole spectrum and the LH fluctuations becomes more important than the RH ones above $\sim 0.1 f_{c i}$. For $\beta \sim 0.5$ and $T_{\perp} / T_{\|} \simeq 3$, the spectral features appear: left and right hand Alfvénic fluctuations form two maxima: around $0.3 f_{c i}$ and around $\sim 0.7 f_{c i}$, and the spectrum of LH fluctuations is dominant. These two spectral maxima have been explained as due to the proton cyclotron and the helium cyclotron modes (Gary et al., 1994a).

It was shown, as well, that the amplitude of the compressible magnetic fluctuations $\delta B_{\|} / B_{0}$ increases clearly with $\beta$. Regarding Alfvénic fluctuations in the magnetosheath, Anderson et al. (1994) show that their amplitude does not increases significantly with $\beta$, and in general it is small $\delta B_{\perp} \ll B_{0}$.

In a statistical study (Czaykowska et al., 2001), 132 samples of magnetosheath fluctuations are analyzed just downstream of quasi-parallel and quasi-perpendicular bowshocks. In this study, 4-min time periods centered at 3, 5 and 7 min from the shock crossing have been analyzed. It was shown that downstream of a quasi-parallel bow-shock for $f<f_{c i}$, the mean magnetosheath turbulent spectra of magnetic components follow an $f^{-0.8}$ power law. Downstream of a quasi-perpendicular bow-shock these spectra follow an $f^{-1.1}$ power law, but it is not very well defined: there are some enhancements in the left-hand Alfvénic fluctuations. As it is described above, in the solar wind the spectrum $\sim f^{-1}$ is observed in the frequency rang below the Kolmogorov's like cascade. This " $1 / f$-noise" (Matthaeus and Goldstein, 1986) is usually attributed to a mixture of uncorrelated Alfvén waves propagating from the Sun (see the discussion in Sect. 1.1). Similar arguments can explain the power law observed in the magnetosheath: it can be due to a mixture of the uncorrelated Alfvén and mirror waves, generated by the anisotropic ion distributions as is discussed above. Note, that these results are valid only for the region just downstream of the bow-shock. The universality of $f^{-1}$ spectrum in the magnetosheath should be verified, we will discuss this point more in details in Sect. 4.

In the statistical study (Czaykowska et al., 2001), the authors consider not only frequencies below $f_{c i}$, but also the high-frequency part $f>f_{c i}$. Regardless of the fact that the mean fluctuation level downstream of a quasi-parallel shock is higher than downstream of a quasi-perpendicular shock, it was shown that both spectra have a break at the vicinity of $f_{c i}$ and that the high-frequency parts of the spectra $f>f_{c i}$ follow the same power-law $\sim f^{-2.6}$. The observed independence of the power-law on the background parameters, like the geometry of the shock, is the first step toward the descrip- tion of the magnetosheath fluctuations in terms of turbulence, that is, a universal phenomenon.

The mean spectrum within the high-frequency part of the magnetosheath turbulence is close to the one observed in the solar wind. But, contrary to what happens in the solar wind, this spectrum $\sim f^{-2.6}$ is observed immediately above the range of uncorrelated waves $\sim f^{-1}$, without the Kolmogorov's like inertial range.

However, the most striking difference between these two type of MHD turbulence is the presence of Alfvén vortices in the magnetosheath at scales comparable to that where the spectral break occurs (Alexandrova et al., 2006). They appear as a large local maximum (or as a spectral knee) at the break of the turbulent spectrum. A similar spectral maximum was observed during a flux transfer event on the magnetopause (Rezeau et al., 1993). This spectral knee has also been observed downstream of quasi-perpendicular bowshocks (Czaykowska et al., 2001) when the upstream solar wind beta was $\beta<0.5$. It was interpreted as a signature of AIC waves. Thanks to the space resolution of Cluster satellites, we could establish that the spectral knee is in fact due to localized structures in the form of Alfvén vortices, and not to AIC plane waves (Alexandrova et al., 2006).

An Alfvén vortex (Petviashvili and Pokhotelov, 1992) is the cylindrical analogue of the non-linear Alfvén wave. In the simplest case, it is a solution of the equations of incompressible ideal magnetohydrodynamics. The qualitative difference with the usual Alfvén wave is that such vortex propagates in a plane nearly perpendicular to the unperturbed magnetic field with a velocity determined by the projection of this field $\mathbf{B}_{0}$ on the plane perpendicular to the vortex axis. Moreover, the well known relation

$\delta V_{\perp} / V_{A}= \pm \delta B_{\perp} / B_{0}$

( $V_{A}=B_{0} / \sqrt{4 \pi \rho}$ being the Alfvén speed), between the perpendicular magnetic field and velocity fluctuations in the Alfvén wave, is replaced for the vortex by a more general relation

$\delta V_{\perp} / V_{A}=\xi \delta B_{\perp} / B_{0}$,

where $\xi$ is determined by the vortex velocity

When the vortex axis is strictly parallel to the field, this velocity is equal to zero. The Alfvén vortices observed in the magnetosheath have their axis almost parallel to the mean magnetic field $\mathbf{B}_{0}$, and therefore move slowly with respect to the ambient plasma.

The presence of such non-linear structures in the magnetosheath can play an important role in the dynamics of turbulence. If, up to now, the small amplitude fluctuations in the magnetosheath were considered as weak turbulence, the observation of Alfvén vortices (Alexandrova et al., 2006) shows the importance of a revision of this classical approach of the magnetosheath turbulence.

In the following of the present paper we analyze first the spectral properties of the magnetosheath fluctuations during 


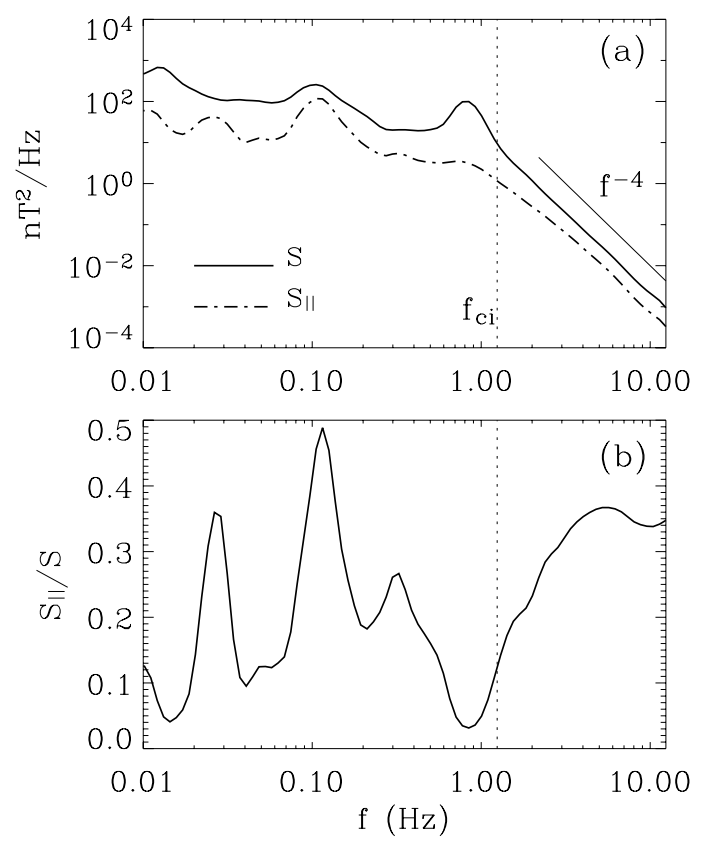

Fig. 1. Spectral properties of magnetic fluctuations in the magnetosheath downstream of a quasi-perpendicular bow-shock during a time period [18:36-18:44] UT on 31 March 2001: (a) Solid line: the total power spectral density of magnetic fluctuations $\mathcal{S}$; dashed line: the spectrum of parallel fluctuations of magnetic field $\mathcal{S}_{\|}$. The straight line refers to a fit with a power law $\propto f^{-4}$. The vertical dotted line indicates the ion cyclotron frequency $f_{c i}=1.2 \mathrm{~Hz}$. (b) The level of compressible fluctuations $S_{\|} / S$ for different frequencies.

a period when Alfvén vortices are observed (Sect. 2). Second, we analyze the spectral properties of the Alfvén vortex solutions (Sect. 3). Finally we propose a model of an Alfvén vortex network that allows to explain several features of the observed magnetosheath spectrum.

\section{Magnetosheath spectrum in the presence of Alfvén vortices}

We consider a time period [18:36-18:44] UT on 31 March 2001, when Cluster was in the magnetosheath downstream of a quasi-perpendicular bow-shock. The average magnetic field (with $4 \mathrm{~s}$ time resolution) is determined from the fluxgate magnetometer (FGM) (Balogh et al., 2001). The ion density $n_{i}$, the plasma bulk velocity $V$ and the ion temperature $T_{i}$ are provided by CIS/HIA instrument (Rème and et al., 2001). The mean plasma parameters are $B_{0}=82 \mathrm{nT}, n_{i}=35 \mathrm{~cm}^{-3}, V=265 \mathrm{~km} / \mathrm{s}, T_{i}=350 \mathrm{eV}$, the Alfvén speed $V_{A}=310 \mathrm{~km} / \mathrm{s}$, the ion cyclotron frequency $f_{c i}=1.2 \mathrm{~Hz}$, the ion inertial length $c / \omega_{p i}=40 \mathrm{~km}$, the ion gyroradius $\rho_{i}=23 \mathrm{~km}, \beta_{i}=0.7$ and ion temperature anisotropy $T_{\perp} / T_{\|}=1.9$. All the data are from Cluster-3 spacecraft.
Magnetic field fluctuations are measured by the search coils (SC) of the STAFF experiment with $0.04 \mathrm{~s}$ time resolution (Cornilleau-Wehrlin et al., 2003). This instrument measures magnetic fluctuations above $0.1 \mathrm{~Hz}$. We merged FGM with STAFF-SC data to obtain a magnetic spectrum covering a large frequency domain (Alexandrova et al., 2004).

Figure 1a shows the total power spectrum density of the magnetic fluctuations $\mathcal{S}=\sum_{i=x, y, z} \mathcal{S}_{i}$ (solid lines) and the spectrum of parallel fluctuations of magnetic field $\mathcal{S}_{\|}$ (dashed-dotted lines). The power spectral density of the magnetic field components is calculated using the Morlet wavelet transform (Torrence and Compo, 1998),

$\mathcal{S}_{i}\left[\mathrm{nT}^{2} / \mathrm{Hz}\right]=\frac{2 \delta t}{N} \sum_{j=0}^{N-1}\left|\mathcal{W}_{i}\left(\tau, t_{j}\right)\right|^{2}$

where

$\mathcal{W}_{i}(\tau, t)=\sum_{j=0}^{N-1} B_{i}\left(t_{j}\right) \psi\left[\left(t_{j}-t\right) / \tau\right]$

is a wavelet coefficient of the $i$-th component of the magnetic field $B_{i}\left(t_{j}\right)$, a data time series with equal time spacing $\delta t$ and $j=0, \ldots, N-1$. Here $\tau$ is a time scale (the corresponding frequency is $f=1 / \tau$ ), while $\psi(u)=\pi^{-1 / 4} \exp \left(-i \omega_{0} u\right) \exp \left(-u^{2} / 2\right)$ is the Morlet wavelet, where $\omega_{0}=6$.

The spectrum of parallel fluctuations $\mathcal{S}_{\|}$is approximated by the spectrum of fluctuations of the magnetic field intensity. This is a correct representation when the amplitudes of the fluctuations verify $\delta B<B_{0}$, which is the case in the magnetosheath downstream of a quasi-perpendicular shock and especially for plasma conditions when $\beta<1$ (see our discussion in Sect. 1).

From Fig. 1a, one can see that the high frequency part of the spectrum, within the range $[2.0,12.5] \mathrm{Hz}$, follows a well defined power law $f^{-4}$. This power law is rather far from the mean spectrum $\sim f^{-2.6}$ determined in (Czaykowska et al., 2001), which we have discussed in Sect. 1. At the lower frequencies, there is no a well-defined powerlaw, but some particular activity is observed. Within the frequency range $[0.01,0.25] \mathrm{Hz}$ the growth of linear waves, AIC and mirror, probably takes place. Within the frequency range $[0.25,0.5] \mathrm{Hz}$, just below a large spectral maximum, we observe a plateau. The spectral maximum, or the spectral knee, is a signature of the Alfvén vortices (Alexandrova et al., 2006), here it covers the $[0.5,2.0] \mathrm{Hz}$ spectral range, i.e. $[0.4,1.6] f_{c i}$.

Figure $1 \mathrm{~b}$ shows the level of compressible fluctuations, i.e. the ration $S_{\|} / S$, as a function of frequency. One can see that the level of compressibility is rather important within the high frequency part of the spectrum. This is the case in the solar wind as well (Alexandrova et al., 2008). Around $0.1 \mathrm{~Hz}$, $S_{\|} / S$ reaches 0.5 , which means equipartition between the energies of compressible and transverse fluctuations. This peak 
can correspond to a mirror mode. At the same time, within the range of the Alfvén vortices $[0.5,2.0] \mathrm{Hz}, S_{\|} / S$ decreases to a negligibly small value 0.03 , reflecting the incompressible nature of the vortices. It seems that the appearance of the Alfvén vortices in a finite beta plasma (here $\beta \sim 1$ ) makes it incompressible within the vortices. A statistical study over $\sim 30$ magnetosheath samples shows (i) a systematic decrease of $S_{\|} / S$ within the spectral knee range, and (ii) Alfvén vortices are observed for not too large $\beta, \beta<3$ (Alexandrova et al., in preparation ${ }^{4}$ ).

\section{The Alfvén vortex and its spectral properties}

The Alfvén vortices are multi-scale nonlinear structures and one may wonder how they can influence the turbulent spectrum (M. Berthomier, private communication, 2006), even outside the observed spectral "knee".

In this section, we begin by a short review of the main theoretical features of the model of incompressible Alfvén vortex (Petviashvili and Pokhotelov, 1992; Kadomtsev and Pogutse, 1974), since it is not so well known in the space physics community. Second, we analyze the spectra of two topologically independent vortex solutions, monopole and dipole. Finally, we discuss the spectral properties of a periodic vortex network.

\subsection{Alfvén vortex solutions}

The Alfvén vortex is one of the non-linear solutions of the ideal incompressible MHD equations. It is characterized by magnetic field and velocity fluctuations mostly perpendicular to the unperturbed magnetic field $\mathbf{B}_{0}$ (taken here as parallel to the $z$ direction), $\delta B_{z} \ll \delta B_{\perp}$ and $\delta V_{z} \ll \delta V_{\perp}$; they have a slow time dependence, $\partial_{t} \ll \Omega_{c i}$, and their space variations verify $\partial_{z} \ll \nabla_{\perp}$. Their amplitude $\varepsilon \sim \delta B_{\perp} / B_{0}$ is assumed to be small although finite, $0<\varepsilon<1$ and they satisfy the following scaling relations:

$$
\frac{\partial_{z}}{\nabla_{\perp}} \sim \frac{\partial_{t}}{V_{A} \nabla_{\perp}} \sim \frac{\delta B_{z}}{\delta B_{\perp}} \sim \frac{\delta V_{z}}{\delta V_{\perp}} \sim \frac{\delta B_{\perp}}{B_{0}} \sim \frac{\delta V_{\perp}}{V_{A}} \sim \varepsilon .
$$

The transverse fluctuations can then be described by two scalar functions, the parallel component of the vector potential $A_{z}$ and a flux function $\psi$

$$
\delta \mathbf{B}_{\perp}=\nabla A_{z} \times \mathbf{z}, \quad \delta \mathbf{V}_{\perp}=\mathbf{z} \times \nabla \psi
$$

(in the following the symbol $\delta$ will be omitted).

For the scalar variables $A_{z}$ and $\psi$ the MHD equations

$$
\begin{array}{r}
\rho\left(\partial_{t}+\mathbf{V} \cdot \nabla\right) \mathbf{V}=-\nabla p+\frac{1}{4 \pi}(\nabla \times \mathbf{B}) \times \mathbf{B} \\
\partial_{t} \mathbf{B}=\nabla \times(\mathbf{V} \times \mathbf{B}) \\
\nabla \cdot \mathbf{V}=0 ; \quad \nabla \cdot \mathbf{B}=0
\end{array}
$$

\footnotetext{
${ }^{4}$ Alexandrova, $\mathrm{PhD}$ thesis, 2005.
}

reduce to two non-linear scalar equations (Kadomtsev and Pogutse, 1974; Strauss, 1976; Petviashvili and Pokhotelov, 1992), the conservation of the momentum along $z$

$\partial_{t} \nabla_{\perp}^{2} \psi+\left\{\psi, \nabla_{\perp}^{2} \psi\right\}=\frac{1}{4 \pi \rho}\left\{A_{z}, \nabla_{\perp}^{2} A_{z}\right\}-\frac{B_{0}}{4 \pi \rho} \partial_{z} \nabla_{\perp}^{2} A_{z}$

and the Maxwell-Faraday equation in the plane perpendicular to $z$

$\partial_{t} A_{z}+B_{0} \partial_{z} \psi+\left\{\psi, A_{z}\right\}=0$.

Here the notation $\{.,$.$\} corresponds to the Poisson bracket (or$ the Jacobian)

$\{a, b\}=\partial_{x} a \partial_{y} b-\partial_{y} a \partial_{x} b \equiv(\nabla a \times \nabla b) \cdot \mathbf{z}$.

These equations can be written in dimensionless form, using new variables $t=\Omega_{c i} t, r_{\perp}=r_{\perp} / \rho_{i}, z=z /\left(c / \omega_{p i}\right)$, $\rho=\rho / \rho_{0}, \Phi=\psi /\left(\rho_{i}^{2} \Omega_{c i}\right), A=A_{z} V_{A} /\left(B_{0} \rho_{i}^{2} \Omega_{c i}\right)$

$d_{t} \nabla_{\perp}^{2} \Phi=\{A, J\}-\partial_{z} J$

$d_{t} A+\partial_{z} \Phi=0$

where $J=\nabla_{\perp}^{2} A$ is the longitudinal current and

$d_{t} \equiv \partial_{t}+\mathbf{V}_{\perp} \cdot \nabla_{\perp}$.

The Alfvén vortices are solutions which are localized in a plane nearly perpendicular to $z$ and propagate with a speed $u$ in this plane while conserving their shape. Choosing the variables in the vortex plane $x$ and $\eta$, with

$\eta=y+\alpha z-u t, \quad \alpha=\tan (\vartheta)$,

$\vartheta$ being the angle between the normal to the plane $(x, \eta)$ and $\mathbf{B}_{0}$, we arrive to a two dimensional problem. In the new variables $(x, \eta)$ the Eqs. (13) and (14) become

$$
\begin{array}{r}
\left\{\Phi-u x, \nabla_{\perp}^{2}(\Phi-u x)\right\}=\{A-\alpha x, J\} \\
\{\Phi-u x, A-\alpha x\}=0
\end{array}
$$

with the new Poisson bracket $\{a, b\}=\partial_{x} a \partial_{\eta} b-\partial_{\eta} a \partial_{x} b$. Equation (17) means that $(\Phi-u x)$ and $(A-\alpha x)$ are dependent on one another:

$A-\alpha x=f(\Phi-u x)$

so that Eq. (16) leads to an equation for $(\Phi-u x)$

$\nabla_{\perp}^{2}(\Phi-u x)=f^{\prime}(\Phi-u x) J+f_{1}(\Phi-u x)$,

containing two arbitrary functions, $f$ and $f_{1}$. There is, therefore, an infinite number of solutions of the system (16) and (17) in the form of vortices.

Among this infinite set of solutions, the simplest Alfvén vortex solution is localized in a circle of the radius $a$ in the plane $(x, \eta)$, and decays at infinity as a power law. It satisfies a generalized Alfvén relation

$\Phi=\xi A, \quad$ with $\xi=\frac{u}{\alpha}$ 


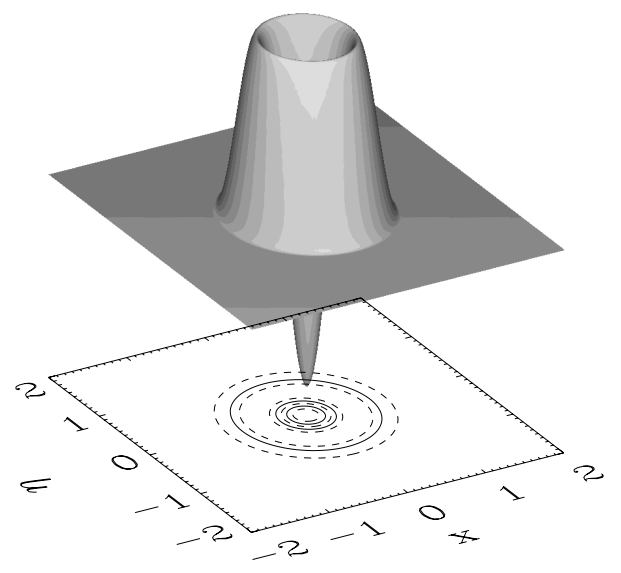

Fig. 2. The surface of the current $J$ above the vortex plane $(x, \eta)$ and the contours of the potential $A$ (that coincide here with the field lines) in this plane for the monopolar structure with the radius of localization $a=1$ and angle $\alpha=0$.

where $\alpha$ and $u$ can be zero only simultaneously. Its current density $J$ is a linear function of $A-\alpha x$ inside a circle of radius $a$ and vanishes outside

$$
\left\{\begin{array}{l}
J=-k^{2}(A-\alpha x-c), \quad r<a \\
J=0, \quad r \geq a
\end{array}\right.
$$

where $k$ and $c$ are constants. This solution is

$$
\left\{\begin{array}{l}
A=A_{0}\left(J_{0}(k r)-J_{0}(k a)\right)-\frac{2 \alpha x}{k r} \frac{J_{1}(k r)}{J_{0}(k a)}+\alpha x, r<a \\
A=a^{2} \frac{\alpha x}{r^{2}}, r \geq a .
\end{array}\right.
$$

Here $A_{0}$ is a constant amplitude, $J_{0}$ and $J_{1}$ are the Bessel functions of 0th and 1st order respectively, $r=\sqrt{x^{2}+\eta^{2}}$ is the radial variable in the plane of the vortex.

The continuity of the solution (22) in $r=a$ requires that the parameter $k$ and the radius $a$ be coupled by the following dispersion relation

$$
J_{1}(k a)=0 \text {. }
$$

This relation ensures the continuity of the magnetic field $\mathbf{B}_{\perp}=\left(B_{x}, B_{\eta}\right)=\left(\partial_{\eta} A,-\partial_{x} A\right)$ in $r=a$ as well as a vanishing divergence of $\mathbf{B}_{\perp}$ everywhere.

Going back to the 3-D problem we must respect the following conditions: since $\partial_{z} \ll \nabla_{\perp}$ has to be satisfied, the angle must be small, $\alpha \sim \partial_{z} / \nabla_{\perp} \sim \varepsilon$. Similarly, the velocity $u$ must be also small in order to satisfy the condition $\partial_{t} \ll \Omega_{c i}$, i.e. $u \sim \partial_{t} / \Omega_{c i} \sim \varepsilon$. In principle, $\xi$ is arbitrary, but of the order of 1 .

The Alfvén vortex solution (22) is the analogue of the incompressible unmagnetized hydrodynamic vortex solution, and as in hydrodynamics, we distinguish here to types of vortices: monopole and dipole.

The monopolar vortex solution correspond to the case with $\alpha=0(u=0)$, i.e., when the projection of the mean field to

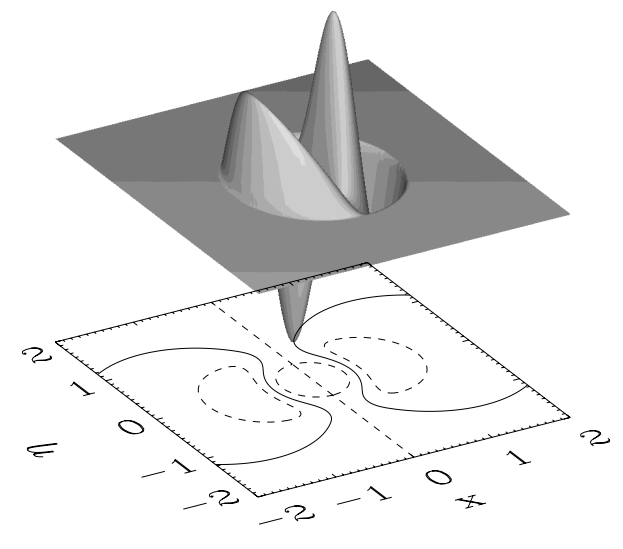

Fig. 3. The same as Fig. 2 but for the bipolar vortex structure with $a=1, \alpha=5^{\circ}$, here the current and field lines are symmetric with respect to the line $x=0$ as far as the amplitude of the monopolar part of the vortex is chosen to be $A_{0}=0$.

the vortex plane is zero. This vortex is at rest in the plasma frame. It corresponds to a field-aligned force-free current localized within a circle of the radius $a$

$$
\left\{\begin{array}{l}
A=A_{0}\left(J_{0}(k r)-J_{0}(k a)\right), \quad r<a \\
A=0, \quad r \geq a
\end{array}\right.
$$

The monopole has the current $J$ and the field lines as is shown in Fig. 2. The contours of its magnetic field components are shown in Fig. 4 (upper panels).

As soon as $\alpha \neq 0(u \neq 0)$, the general solution (22) describes the dipolar vortex. It is not stationary in the plasma as the monopole, but propagates with velocity $u$ along the $\eta$ direction, the direction of the mean field projection on the vortex plane. The current of the dipolar vortex and its field lines are presented in Fig. 3. Here the amplitude of monopolar part $A_{0}$ is chosen to be zero, otherwise $A, J$ and the magnetic field lines are no more symmetric with respect to the vortex center. The contours of its magnetic field components are shown in Fig. 4 (lower panels).

Thus monopolar and dipolar vortices are topologically different and there is no continuous transition between them. These differences reflect themselves in the Fourier spectra of these two vortex types.

\subsection{Power spectra of monopole and dipole}

Suppose now that a magnetic probe moves in space, along the $\mathrm{x}$-axis with a constant velocity and a distance of closest approach to the vortex axis $\eta$. Figure 5 (upper panels) shows the "measured" $B_{x}$-profiles of monopole and dipole vortex structures, for $\eta=-0.2 a$. The lower panels of Fig. 5 show the power spectral densities (PSD) of these signals calculated via Fourier (solid lines) and via the Morlet Wavelet Transforms (empty circles). The power spectra of both, monopole and dipole, have a knee around the wave vector $k=1$, 

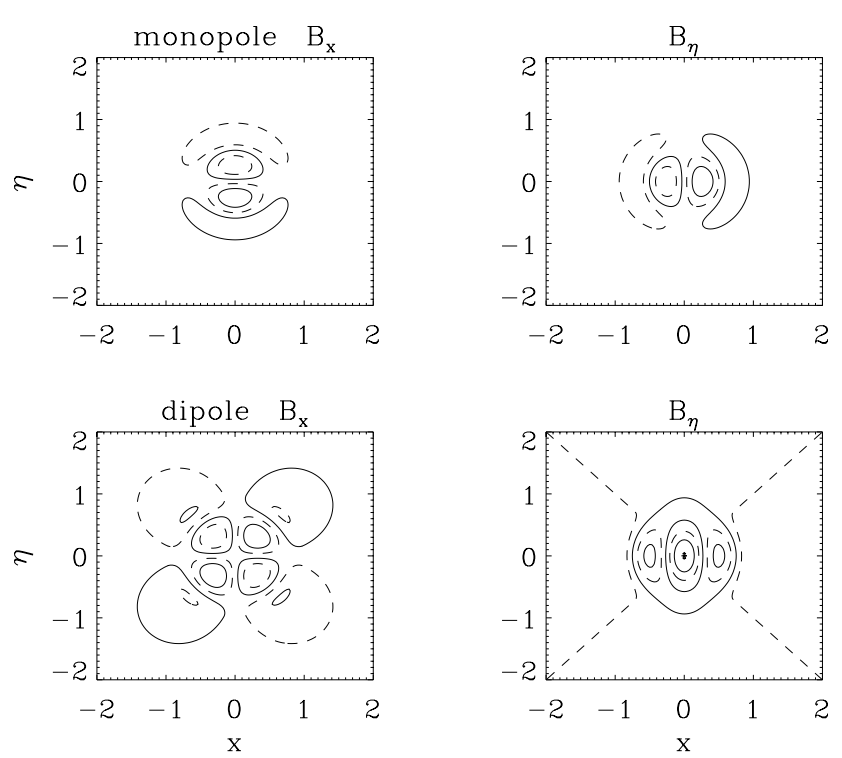

Fig. 4. Upper panels: contours of the magnetic field components, $B_{x}$ and $B_{\eta}$, in a monopolar vortex structure with $a=1$ and $\alpha=0$. Lower panels: contours of $B_{x}$ and $B_{\eta}$ in a dipolar vortex with $a=1$, $\alpha=5^{\circ}$ and $A_{0}=0$.

corresponding to a vortex radius $a=1$. Above this maximum, for $k>2$, well-defined power laws are observed. The monopole vortex spectrum follows $\sim k^{-4}$, while the dipolar follows $\sim k^{-6}$. These power laws can be easily explained.

The magnetic field of a monopole vortex is completely localized within the circle of the radius $a$. It yields to a discontinuity at $r=a$ for the current density, thus the PSD of $J$ follows a $k^{-2}$-power law. Therefore the power spectral density of the magnetic field components is $k^{-4}$. In the case of a dipolar vortex structure, the current is localized while the field extends to infinity. Therefore the PSD of the derivative of the current follows a $k^{-2}$-law, that of the current is $\propto k^{-4}$ and that of the field follows a $k^{-6}$-law.

Note that these spectra are not completely independent of the trajectory of the virtual probe through the vortices. Along some particular trajectories, the magnetic field components are equal to zero and then the spectrum vanishes. These trajectories are vortex separatrices, which can be easily seen in Fig. 4. For example, for the monopole structure, the trajectory along $\mathrm{x}$-axis with $\eta=0$ is a separatrix of the $B_{x}$ component, and the one along $\eta$ with $x=0$ is a separatrix of $B_{y}$. Dipole has twice as many separatrices: the trajectory along x-axis with $\eta=0$ and the one along $\eta$ with $x=0$ are the separatrices of $B_{x}$, while $B_{y}=0$ along the diagonals. Actually, the probability that the satellite crosses the vortex along a separatrix is small and the spectra of Fig. 5 can be considered as quasi-universal.

The vortex spectra presented above can partially explain the magnetosheath magnetic spectrum presented in Fig. 1.
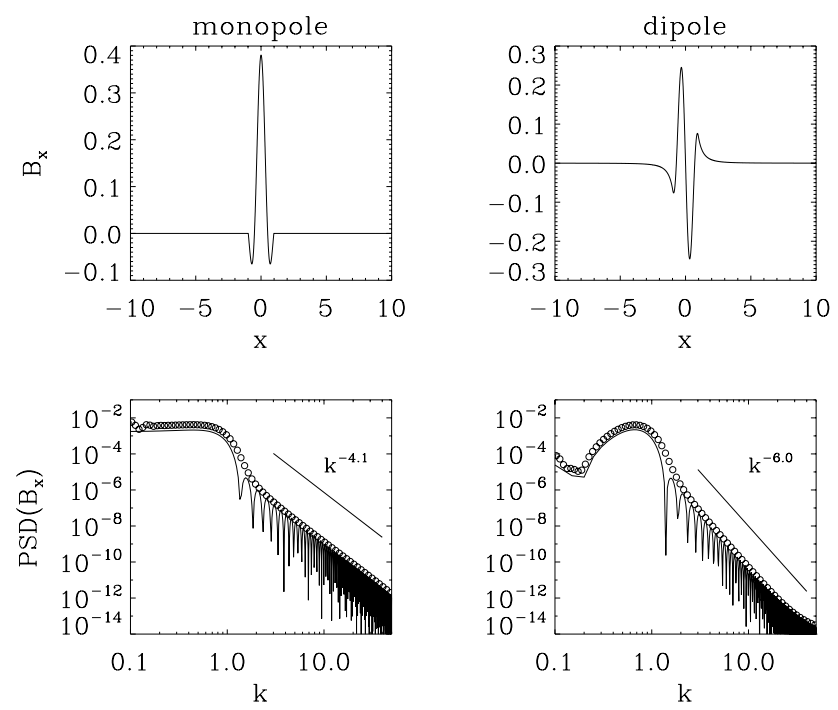

Fig. 5. Upper panels: $B_{x}$ component of the magnetic field of the monopole (left) and dipole (right) measured along $x$ with $\eta=-0.2 a$. Lower panels: the Fourier (solid line) and the Morlet (empty circles) transformations of the signals of the upper panels. The straight lines refer to the power law fits.

First, both vortex models reproduce the spectral knee, it appears to be around $k=a^{-1}$. Second, the rather steep power laws of the monopole and dipole structures can explain the important steepening of the magnetosheath spectrum. In a real plasma one expects that the vortex singularities, which give the power law spectra, will be regularized by dispersion or dissipation effects at some scale $\ell_{r}$. That means that at smaller scales, $\ell<\ell_{r}$, the spectrum will follow an exponential law and no longer a power law. We think that this regularization happens at electron scales, $\ell_{r} \simeq \lambda_{e}$. Doppler shifted electron inertial length $\lambda_{e} \simeq 5 \mathrm{~km}$ appears in the magnetosheath spectrum at around $f \sim 50 \mathrm{~Hz}$, well outside of the analyzed frequency range, see Fig. 1a.

Now, let us discuss a model, which affects the wave vectors below the spectral maximum.

\subsection{Vortex network}

The observed magnetosheath spectrum of Fig. 1 is the result of an integration over a number of events, and particularly over an important number of Alfvén vortex structures. We suggest, via the model explained below, that Cluster crosses an Alfvén vortex network, as is shown in Fig. 6.

Figure 7 (upper panels) shows the $B_{x}$ component of the magnetic field measured along a trajectory $r$ through a monopolar (left) and a dipolar (right) regular vortex network. The distance between two successive vortices - network parameter - is chosen to be $\lambda=10 a$ in both cases.

Figure 7 (lower panels) shows the wavelet spectra of the upper panel signal. The spectrum of the monopolar vortex network is similar to the spectrum of Fig. 5 (left) describing 


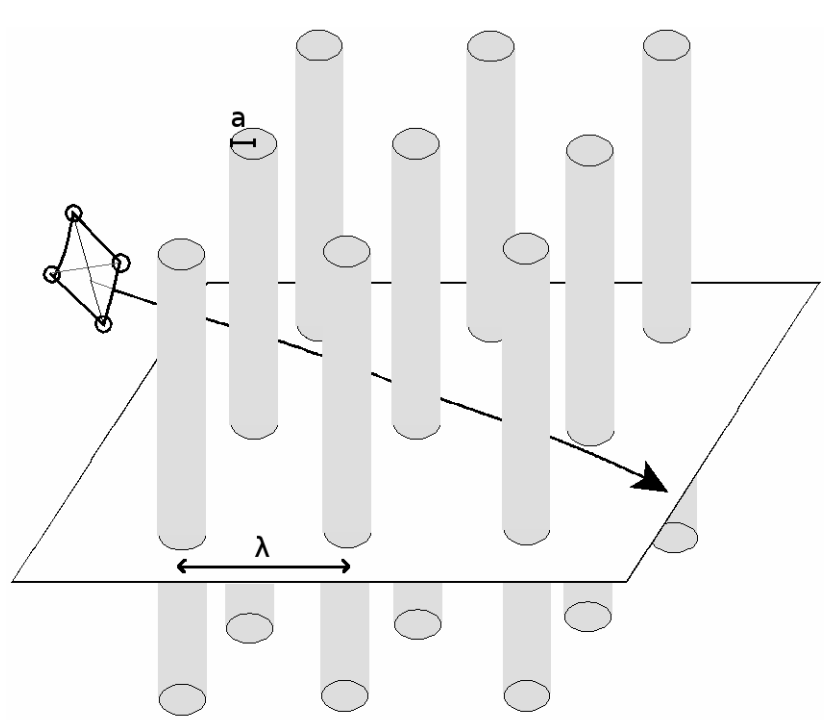

Fig. 6. Cartoon of a regular network of Alfvén vortices of radius $a$, two successive vortices are separated by a distance $\lambda$.

the single vortex case, but now there is a plateau between the network parameter $\lambda^{-1}$ and the vortex scale $a^{-1}$.

The spectrum of the dipolar vortex network is $\sim k^{-2}$ for the wave vectors $k \geq 10$. Small scales discontinuities of the field and non-periodicity of the signal are at the origin of this slope. In the real infinite plasma at high wave vectors we expect to observe the spectrum $k^{-6}$ of a single dipolar vortex, shown in Fig. 5. At lower $k$ there is a plateau between $\lambda^{-1}$ and $a^{-1}$ scales, as for the monopolar network case.

A similar plateau is observed in the magnetosheath spectrum of Fig. 1 for the frequency range $[0.25,0.5] \mathrm{Hz}$, while the spectral knee is centered around $1 \mathrm{~Hz}$. The model shown above allows the determination of $a$ and $\lambda$ from the spectral shape only. Taking into account the Taylor hypothesis, from the spectral shape of Fig. 1 it follows that $\lambda \simeq 1000 \mathrm{~km}$ $\left(\simeq 26 c / \omega_{p i}\right)$ and the vortex radius is $a \simeq 300 \mathrm{~km}\left(\simeq 8 c / \omega_{p i}\right)$. The same value for the mean separation between the vortices $\langle\lambda\rangle \simeq 1000 \mathrm{~km}$ obtains if one assumes an irregular network.

\section{Discussion and conclusion}

Let us summarize now some common and distinctive features of the plasma turbulence in the two natural laboratories considered here. In the solar wind as in the magnetosheath the fluctuations are anisotropic with respect to the mean field $\mathbf{B}_{0}$ : the smaller the plasma beta, the higher the anisotropy $\delta B_{\perp}>\delta B_{\|}$(Bruno and Carbone, 2005; Anderson et al., 1994). In both regions the anisotropy in wave vector distributions, $k_{\perp}>k_{\|}$, is observed (Bieber et al., 1996; Horbury et al., 2005; Sahraoui et al., 2006; Mangeney et al., 2006; Osman and Horbury, 2007). In both regions the turbu-
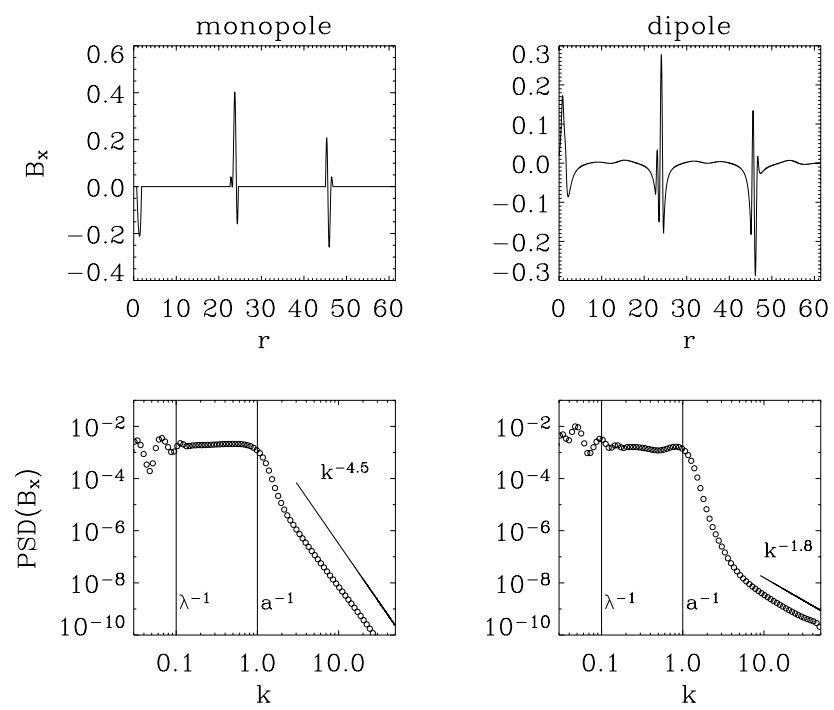

Fig. 7. Upper panels: $B_{x}$ measured along the trajectory $\mathbf{r}$ through the network of the monopolar (left) and dipolar (right) vortex structures. Lower panels: power spectral density of the signals of the upper panels calculated using the Morlet wavelet transform. Vertical lines indicate the network parameter $\lambda^{-1}$ and the vortex scale $a^{-1}$.

lent spectrum has a break at the vicinity of the ion characteristic scales (Leamon et al., 1998; Czaykowska et al., 2001).

Contrary to what happens in the solar wind, in the magnetosheath the mean spectrum follows $\sim f^{-1}$ below the spectral break and $\sim f^{-2.6}$ above it, without the Kolmogorov's-like inertial range (Czaykowska et al., 2001). The universality of this spectrum, however, should be verified. In the solar wind, the Kolmogorov's like inertial range is developed as far as the non-linear time of the interactions $\tau_{\mathrm{NL}}$ is shorter that the transit time. The shortest transit time in the magnetosheath (along the Sun-Earth line) is of the order of few minutes. For example, for the AIC wave studied in (Alexandrova et al., 2004 ) with the wave length $\lambda \sim 600 \mathrm{~km}$ and the amplitude of the velocity fluctuations $\delta V \sim 30 \mathrm{~km} / \mathrm{s}$, the non-linear time is estimated to be $\tau_{\mathrm{NL}} \sim 20$ s, i.e. smaller than the transit time. That means that the development of the Kolmogorov's-like inertial range is possible in the magnetosheath, especially on the flanks where the transit time is longer.

Another difference between these two natural laboratories, and the most important from our point of view, is the presence of the incompressible Alfvén vortices in the compressible magnetosheath plasma. Even for $\beta \sim 1$, the plasma seems to be incompressible within the vortices. Their appearance in the magnetosheath can be the result of some non-linear evolution of the fluctuations of the $f^{-1}$ spectrum.

Are the Alfvén vortices inherent only to the downstream region of the shocks? For neutral fluids it was shown that the curvature of the shock injects vorticity downstream (Kevlahan, 1997). Similar effect can appear in the case of 
collisionless MHD shock waves such as the Earth's bowshock. The scale of the vortices generated via such process is related to the typical radius of curvature. So the large scale shock curvature can be at the origin of vortices at scales of the order of some Earth's radius $R_{E}$. The Earth bow shock is actually unsteady and small scale ripples propagate along the shock front, which may be at the origin of the observed Alfvén vortices. This topics requires a detailed investigation which has yet to be done.

Are there Alfvén vortices in the solar wind? For instance, there is no solar wind observation that exhibits the Alfvén vortex signature at the spectral break point like in the magnetosheath. However, at larger scales, some signatures of the magnetic vortices are observed. Magnetic vortices with a cross-section of the order of $0.3 \mathrm{AU}$ can appear as a result of the interaction between fast and slow solar wind flows (Drillia and Moussas, 1996). Verkhoglyadova et al. (2003) analyzed magnetic discontinuities in the solar wind and proposed to explain them in terms of Alfvén vortices. Multisatellite analysis, however, is needed to confirm this conclusion.

In the magnetospheric plasma sheet, where $\beta \sim 1$ and $\mathbf{B}_{0}$ is mostly perpendicular to the plasma flow, Verkhoglyadova et al. (1999) observe the signatures of Alfvén vortices within the regions where magnetic pressure is in antiphase with plasma pressure. That can be a result of a focusing of an Alfvén wave propagating in a plasma with density channels (T. Passot, private communication, 2007).

In a strongly inhomogeneous plasma with a plasma beta $m_{e} / m_{i} \ll \beta \sim\left(\rho_{i} \omega_{p i} / c\right)^{2} \ll 1$ drift Alfvén vortices appear (Horton and Hasegawa, 1994). Their cross-section $a$ is of the order of the ion Larmor radius $\rho_{i}$ and much smaller than the ion-inertial length $c / \omega_{p i}$. The observations of such vortices in the high-latitude ionosphere have been firstly reported by Chmyrev et al. (1988). In the same region, solitary kinetic Alfvén waves have been studied by Volwerk et al. (1996). Using space resolution of Cluster, the drift kinetic Alfvén vortices have been identified in the high-altitude cusp region (Sundkvist et al., 2005). We remark that the drift Alfvén vortices and the MHD vortices discussed in the present paper have similar structure, however the underling physics are different.

In the magnetosheath the signatures of the Alfvén vortices are frequently observed downstream of the quasiperpendicular bow-shock with plasma beta $\beta<3$ (the statistical analysis of strong Alfvénic fluctuations in the magnetosheath is a subject of an incoming paper). When the Alfvén vortices are rather energetic with respect to the surrounding noise, i.e. the maximum on the spectral break is pronounced, the high frequency part of the spectrum becomes steeper than the mean magnetosheath spectrum and flatter at lower frequencies, sometimes a plateau appears.

Here we show that the spectra of the Alfvén vortex solutions follow well-defined power-laws for $k>a^{-1}, a$ being the radius of the vortex. The spectrum of the monopole vortex follows $k^{-4}$-law, due to the discontinuities of its current. In the case of the dipole vortex structure, the current is continuous but the derivative of the current has discontinuity at $r=a$ and so the magnetic spectrum follows $k^{-6}$-law. These spectra are quasi-universal, i.e. they are independent on the satellite trajectory through the vortex. Forbidden trajectories are the vortex separatrices, where the field components are zero and so the spectra are not defined.

Simulating the Alfvén vortex network we recover the observed spectral plateau on the scales between the network parameter $\lambda^{-1}$ and $a^{-1}$. Taking into account the Taylor hypothesis, which is always verified for the convected structures, like field-aligned vortices, the observed anomalous steepening of the high frequency spectrum and the plateau on the lower frequencies can be therefore explained by the spectra of the Alfvén vortex network. Moreover, the spectral break itself and the large maximum on it are predicted by the Alfvén vortex network as well.

We can not affirm, however, that the observed $f^{-4}$ spectrum is due to the presence of the monopole Alfvén vortices only. As is shown in (Alexandrova et al., 2006) both vortex types are present in the magnetosheath turbulence. Moreover the increase of compressible fluctuations observed within the high frequency part of the spectrum can not be explained within the framework of the Alfvén vortex model. Hence the observed spectrum results from a complex of different phenomena, but Alfvén vortices contribute in making the spectrum steeper above the break and flatter below it.

Acknowledgements. O. Alexandrova thanks A. Mangeney, R. Grappin, C. Lacombe and M. Berthomier for fruitful discussions and she acknowledges the support of the French Centre National d'Etude Spatiales (CNES).

Edited by: B. Tsurutani

Reviewed by: two anonymous referees

\section{References}

Alexandrova, O., Mangeney, A., Maksimovic, M., Lacombe, C., Cornilleau-Wehrlin, N., Lucek, E. A., Décréau, P. M. E., Bosqued, J.-M., Travnicek, P., and Fazakerley, A. N.: Cluster observations of finite amplitude Alfvén waves and small-scale magnetic filaments downstream of a quasi-perpendicular shock, J. Geophys. Res., 109, A05207, doi:10.1029/2003JA010056, 2004.

Alexandrova, O., Mangeney, A., Maksimovic, M., CornilleauWehrlin, N., Bosqued, J.-M., and André, M.: Alfvén vortex filaments observed in magnetosheath downstream of a quasiperpendicular bow shock, J. Geophys. Res., 111, A12208, doi: 10.1029/2006JA011934, 2006.

Alexandrova, O., Carbone, V., Veltri, P., and Sorriso-Valvo, L.: Solar wind CLUSTER observations: turbulent spectra and role of Hall effect, Planet. Space Sci., 55, 2224-2227, doi:10.1016/j.pss. 2007.05.022, 2007.

Alexandrova, O., Carbone, V., Veltri, P., and Sorriso-Valvo, L.: Small scale energy cascade of the solar wind turbulence, The Astrophysical Journal, 674, p. 1153, 2008. 
Anderson, B. J., Fuselier, S. A., Gary, S. P., and Denton, R. E.: Magnetic spectral signatures in the Earth's magnetosheath and plasma depletion layer, J. Geophys. Res., 99, 5877-5891, 1994.

Bale, S. D., Kellogg, P. J., Mozer, F. S., Horbury, T. S., and Reme, H.: Measurement of the Electric Fluctuation Spectrum of Magnetohydrodynamic Turbulence, Phys. Rev. Lett., 94, 215002, doi: 10.1103/PhysRevLett.94.215002, 2005.

Balogh, A., Carr, C. M., Acuña, M. H., Dunlop, M. W., Beek, T. J. et al.: The Cluster Magnetic Field Investigation: overview of inflight performance and initial results, Ann. Geophys., 19, 12071217, 2001, http://www.ann-geophys.net/19/1207/2001/.

Bieber, J. W., Wanner, W., and Matthaeus, W. H.: Dominant twodimensional solar wind turbulence with implications for cosmic ray transport, J. Geophys. Res., 101, 2511-2522, doi:10.1029/ 95JA02588, 1996.

Biskamp, D., Schwarz, E., and Drake, J. F.: Two-Dimensional Electron Magnetohydrodynamic Turbulence, Phys. Rev. Lett., 76, 1264-1267, doi:10.1103/PhysRevLett.76.1264, 1996.

Bruno, R. and Carbone, V.: The Solar Wind as a Turbulence Laboratory, Living Reviews in Solar Physics, 2, 4, 186 pp., 2005.

Burlaga, L. F., Wang, C., Richardson, J. D., and Ness, N. F.: LargeScale Magnetic Field Fluctuations and Development of the 19992000 Global Merged Interaction Region: 1-60 AU, The Astrophysical Journal, 585, 1158-1168, doi:10.1086/346108, 2003.

Chevillard, L., Castaing, B., and Lévêque, E.: On the rapid increase of intermittency in the near-dissipation range of fully developed turbulence, European Physical Journal B, 45, 561-567, doi:10. 1140/epjb/e2005-00214-4, 2005.

Chmyrev, V. M., Bilichenko, S. V., Pokhotelov, V. I., Marchenko, V. A., and Lazarev, V. I.: Alfven vortices and related phenomena in the ionosphere and the magnetosphere, Physica Scripta, 38, 841-854, 1988.

Cornilleau-Wehrlin, N., Chanteur, G., Perraut, S., Rezeau, L., Robert, P. et al.: First results obtained by the Cluster STAFF experiment, Ann. Geophys., 21, 437-456, 2003, http://www.ann-geophys.net/21/437/2003/.

Czaykowska, A., Bauer, T. M., Treumann, R. A., and Baumjohann, W.: Magnetic field fluctuations across the Earth's bow shock, Ann. Geophys., 19, 275-287, 2001, http://www.ann-geophys.net/19/275/2001/.

Dmitruk, P., Matthaeus, W. H., and Oughton, S.: Direct comparisons of compressible MHD and reduced MHD turbulence, Physics of Plasmas, 12, 112304, 2005.

Drillia, G. A. and Moussas, X.: Vortices near the Wavy Heliospheric Current Sheet, Sol. Phys., 166, 403-413, 1996.

Fleck Jr., R. C.: Scaling Relations for the Turbulent, Non-Selfgravitating, Neutral Component of the Interstellar Medium, The Astrophysical Journal, 458, 739-741, doi:10.1086/176853, 1996.

Frisch, U.: Turbulence. The legacy of A. N. Kolmogorov, Cambridge, Cambridge University Press, 1995.

Galtier, S.: Wave turbulence in incompressible Hall magnetohydrodynamics, Journal of Plasma Physics, 72, 721-769, 2006.

Galtier, S. and Buchlin, E.: Multiscale Hall-Magnetohydrodynamic Turbulence in the Solar Wind, The Astrophysical Journal, 656, 560-566, doi:10.1086/510423, 2007.

Gary, S. P., Convery, P. D., Denton, R. E., Fuselier, S. A., and Anderson, B. J.: Proton and helium cyclotron anisotropy instability thresholds in the magnetosheath, J. Geophys. Res., 99, 59155921, 1994a.

Gary, S. P., McKean, M. E., Winske, D., Anderson, B. J., Denton, R. E., and Fuselier, S. A.: The proton cyclotron instability and the anisotropy/ $\beta$ inverse correlation, J. Geophys. Res., 99, 5903$5914,1994 b$.

Ghosh, S., Siregar, E., Roberts, D. A., and Goldstein, M. L.: Simulation of high-frequency solar wind power spectra using Hall magnetohydrodynamics, J. Geophys. Res., 101, 2493-2504, doi: 10.1029/95JA03201, 1996.

Grappin, R.: Onset and decay of two-dimensional magnetohydrodynamic turbulence with velocity-magnetic field correlation, Physics of Fluids, 29, 2433-2443, 1986.

Hellinger, P., Trávníček, P., Mangeney, A., and Grappin, R.: Hybrid simulations of the magnetosheath compression: Marginal stability path, Geophys. Res. Lett., 30, 1959, doi:10.1029/ 2003GL017855, 2003.

Hellinger, P., Trávníček, P., Kasper, J. C., and Lazarus, A. J.: Solar wind proton temperature anisotropy: Linear theory and WIND/SWE observations, Geophys. Res. Lett., 33, L09101, doi: 10.1029/2006GL025925, 2006.

Horbury, T. S., Forman, M. A., and Oughton, S.: Spacecraft observations of solar wind turbulence: an overview, Plasma Physics and Controlled Fusion, 47, B703-B717, doi:10.1088/ 0741-3335/47/12B/S52, 2005.

Kadomtsev, B. B. and Pogutse, O. P.: Nonlinear helical perturbations of a plasma in the tokamak, Zhurnal Eksperimentalnoi i Teoreticheskoi Fiziki, 65, 575-589, 1974.

Kevlahan, N. K.-R.: The vorticity jump across a shock in a nonuniform flow, Journal of Fluid Mechanics, 341, 371-384, 1997.

Lacombe, C. and Belmont, G.: Waves in the Earth's magnetosheath: Observations and interpretations, Adv. Space Res., 15, 329-340, 1995.

Lacombe, C., Pantellini, F. G. E., Hubert, D., Harvey, C. C., Mangeney, A., Belmont, G., and Russell, C. T.: Mirror and Alfvenic waves observed by ISEE 1-2 during crossings of the earth's bow shock, Ann. Geophys., 10, 772-784, 1992, http://www.ann-geophys.net/10/772/1992/.

Leamon, R. J., Smith, C. W., Ness, N. F., Matthaeus, W. H., and Wong, H. K.: Observational constraints on the dynamics of the interplanetary magnetic field dissipation range, J. Geophys. Res., 103, 4775-4787, 1998.

Leamon, R. J., Smith, C. W., Ness, N. F., and Wong, H. K.: Dissipation range dynamics: Kinetic Alfvén waves and the importance of electron beta $\beta_{e}$, J. Geophys. Res., 104, 22 331-22 344, doi: 10.1029/1999JA900158, 1999.

Li, H., Gary, S. P., and Stawicki, O.: On the dissipation of magnetic fluctuations in the solar wind, Geophys. Res. Lett., 28, 13471350, doi:10.1029/2000GL012501, 2001.

Lucek, E. A., Dunlop, M. W., Horbury, T. S., Balogh, A., Brown, P., Cargill, P., Carr, C., Fornaçon, K.-H., Georgescu, E., and Oddy, T.: Cluster magnetic field observations in the magnetosheath: four-point measurements of mirror structures, Ann. Geophys., 19, 1421-1428, 2001, http://www.ann-geophys.net/19/1421/2001/.

Mangeney, A., Lacombe, C., Maksimovic, M., Samsonov, A. A., Cornilleau-Wehrlin, N., Harvey, C. C., Bosqued, J.-M., and Trávníček, P.: Cluster observations in the magnetosheath - Part 1: Anisotropies of the wave vector distribution of the turbulence 
at electron scales, Ann. Geophys., 24, 3507-3521, 2006, http://www.ann-geophys.net/24/3507/2006/.

Matsuoka, A., Southwood, D. J., Kokubun, S., and Mukai, T.: Propagation sense of low-frequency MHD waves in the magnetosheath observed by Geotail, J. Geophys. Res., 105, $18361-$ 18 376, doi:10.1029/2000JA900014, 2000.

Matteini, L., Landi, S., Hellinger, P., and Velli, M.: Parallel proton fire hose instability in the expanding solar wind: Hybrid simulations, J. Geophys. Res. (Space Phys.), 111, A10101, doi: 10.1029/2006JA011667, 2006.

Matthaeus, W. H. and Goldstein, M. L.: Low-frequency 1/f noise in the interplanetary magnetic field, Phys. Rev. Lett., 57, 495-498, 1986.

Matthaeus, W. H., Goldstein, M. L., and Roberts, D. A.: Evidence for the presence of quasi-two-dimensional nearly incompressible fluctuations in the solar wind, J. Geophys. Res., 95, $20673-$ $20683,1990$.

Matthaeus, W. H., Ghosh, S., Oughton, S., and Roberts, D. A.: Anisotropic three-dimensional MHD turbulence, J. Geophys. Res., 101, 7619-7630, doi:10.1029/95JA03830, 1996.

Müller, W.-C. and Grappin, R.: Spectral Energy Dynamics in Magnetohydrodynamic Turbulence, Phys. Rev. Lett., 95, 114502, doi: 10.1103/PhysRevLett.95.114502, 2005.

Osman, K. T. and Horbury, T. S.: Multispacecraft Measurement of Anisotropic Correlation Functions in Solar Wind Turbulence, The Astrophysical Journal Letters, 654, L103-L106, doi:10. 1086/510906, 2007.

Petviashvili, V. I. and Pokhotelov, O. A.: Solitary Waves in Plasmas and in the Atmosphere, Gordon \& Breach Science Pub, 1992.

Rème, H., Aoustin, C., Bosqued, J. M., Dandouras, I., Lavraud, B. et al.: First multispacecraft ion measurements in and near the Earth's magnetosphere with the identical Cluster ion spectrometry (CIS) experiment, Ann. Geophys., 19, 1303-1354, 2001, http://www.ann-geophys.net/19/1303/2001/.

Rezeau, L., Perraut, S., and Roux, A.: Electromagnetic fluctuations in the vicinity of the magnetopause, Geophys. Res. Lett., 13, 1093-1096, 1986.

Rezeau, L., Roux, A., and Russell, C. T.: Characterization of smallscale structures at the magnetopause from ISEE measurements, J. Geophys. Res., 98, 179-186, 1993.

Rezeau, L., Belmont, G., Cornilleau-Wehrlin, N., Reberac, F., and Briand, C.: Spectral law and polarization properties of the lowfrequency waves at the magnetopause, Geophys. Res. Lett., 26, 651-654, doi:10.1029/1999GL900060, 1999.

Rezeau, L., Sahraoui, F., D’Humières, E., Belmont, G., Chust, T. et al.: A case study of low-frequency waves at the magnetopause, Ann. Geophys., 19, 1463-1470, 2001, http://www.ann-geophys.net/19/1463/2001/.

Sahraoui, F., Belmont, G., and Rezeau, L.: Hamiltonian canonical formulation of Hall-magnetohydrodynamics: Toward an application to weak turbulence theory, Physics of Plasmas, 10, 1325, 2003a.

Sahraoui, F., Pinçon, J. L., Belmont, G., Rezeau, L., CornilleauWehrlin, N. et al.: ULF wave identification in the magnetosheath: The k-filtering technique applied to Cluster II data, J. Geophys. Res. (Space Phys.), 108, 1335, doi:10.1029/2002JA009587, 2003b.

Sahraoui, F., Belmont, G., Rezeau, L., Cornilleau-Wehrlin, N., Pinçon, J. L., and Balogh, A.: Anisotropic Turbulent Spectra in the Terrestrial Magnetosheath as Seen by the Cluster Spacecraft, Phys. Rev. Lett., 96, 075002, doi:10.1103/PhysRevLett.96. 075002, 2006.

Schwartz, S. J., Burgess, D., and Moses, J. J.: Low-frequency waves in the Earthś magnetosheath: present status, Ann. Geophys., 14, 1134-1150, 1996, http://www.ann-geophys.net/14/1134/1996/.

Shebalin, J. V., Matthaeus, W. H., and Montgomery, D.: Anisotropy in MHD turbulence due to a mean magnetic field, Journal of Plasma Physics, 29, 525-547, 1983.

Shevyrev, N. N. and Zastenker, G. N.: Some features of the plasma flow in the magnetosheath behind quasi-parallel and quasi-perpendicular bow shocks, Planet. Space Sci., 53, 95-102, doi:10.1016/j.pss.2004.09.033, 2005.

Siscoe, G. L., Davis, L., Smith, E. J., Coleman, P. J., and Jones, D. E.: Magnetic Fluctuations in the Magnetosheath: Mariner 4, J. Geophys. Res., 72, p. 1, 1967.

Smith, C. W., Hamilton, K., Vasquez, B. J., and Leamon, R. J.: Dependence of the Dissipation Range Spectrum of Interplanetary Magnetic Fluctuationson the Rate of Energy Cascade, The Astrophysical Journal Letters, 645, L85-L88, doi:10.1086/506151, 2006.

Sorriso-Valvo, L., Carbone, V., Veltri, P., Consolini, G., and Bruno, R.: Intermittency in the solar wind turbulence through probability distribution functions of fluctuations, Geophys. Res. Lett., 26, 1801-1804, doi:10.1029/1999GL900270, 1999.

Stawicki, O., Gary, S. P., and Li, H.: Solar wind magnetic fluctuation spectra: Dispersion versus damping, J. Geophys. Res., 106, 8273-8282, doi:10.1029/2000JA000446, 2001.

Strauss, H. R.: Nonlinear, three-dimensional magnetohydrodynamics of noncircular tokamaks, Physics of Fluids, 19, 134-140, 1976.

Torrence, C. and Compo, G. P.: A Practical Guide to Wavelet Analysis, B. Am. Meteorol. Soc., 79, 61-78, 1998.

Tsurutani, B. T., Smith, E. J., Anderson, R. R., Ogilvie, K. W., Scudder, J. D., Baker, D. N., and Bame, S. J.: Lion roars and nonoscillatory drift mirror waves in the magnetosheath, J. Geophys. Res., 87, 6060-6072, 1982.

Tsurutani, B. T., Lakhina, G. S., Pickett, J. S., Guarnieri, F. L., Lin, N., and Goldstein, B. E.: Nonlinear Alfvén waves, discontinuities, proton perpendicular acceleration, and magnetic holes/decreases in interplanetary space and the magnetosphere: intermediate shocks?, Nonlin. Processes Geophys., 12, 321-336, 2005 , http://www.nonlin-processes-geophys.net/12/321/2005/.

Tu, C.-Y. and Marsch, E.: MHD structures, waves and turbulence in the solar wind: Observations and theories, Space Sci. Rev., 73, $1-2,1995$.

Vasquez, B. J., Abramenko, V. I., Haggerty, D. K., and Smith, C. W.: Numerous small magnetic field discontinuities of Bartels rotation 2286 and the potential role of Alfvénic turbulence, J. Geophys Res. (Space Phys.), 112, A11102, doi:10.1029/2007JA012504, 2007.

Veltri, P.: MHD turbulence in the solar wind: self-similarity, intermittency and coherent structures, Plasma Physics and Controlled Fusion, 41, A787-A795, 1999.

Veltri, P. and Mangeney, A.: Scaling Laws and Intermittent Structures in Solar Wind MHD Turbulence, in: Solar Wind Nine, edited by: Habbal, S. R., Esser, R., Hollweg, J. V., and Isenberg, 
P. A., Vol. 471 of American Institute of Physics Conference Series, p. 543, 1999.

Verkhoglyadova, O., Agapitov, A., Andrushchenko, A., Ivchenko, V., Romanov, S., and Yermolaev, Y.: Compressional wave events in the dawn plasma sheet observed by Interball-1, Ann. Geophys., 17, 1145-1154, 1999,

http://www.ann-geophys.net/17/1145/1999/.
Verkhoglyadova, O. P., Dasgupta, B., and Tsurutani, B. T.: Model for vortex turbulence with discontinuities in the solar wind, Nonlin. Processes Geophys., 10, 335-343, 2003, http://www.nonlin-processes-geophys.net/10/335/2003/.

Volwerk, M., Louarn, P., Chust, T., Roux, A., de Feraudy, H., and Holback, B.: Solitary kinetic Alfvén waves: A study of the Poynting flux, J. Geophys. Res., 101, 13 335-13 344, doi: 10.1029/96JA00166, 1996. 